\title{
Comentario al artículo "Correlación geoquímica entre crudos y rocas del sistema petrolero de la península de Santa Elena y el golfo de Guayaquil" por Lorenzo et al
}

\author{
Antenor Alemán M. ${ }^{*}$, Galo Montenegro ${ }^{2}$, Allly Palencia ${ }^{1}$, Eduardo Lezama ${ }^{1}$
}

DOI: http://dx.doi.org/10.18273/revbol.v41n2-2019009 @ (1)

Forma de citar: Alemán, A., Montenegro, G., Palencia, A., y Lezama, E. (2019). Comentario al artículo "Correlación geoquímica entre crudos y rocas del sistema petrolero de la península de Santa Elena y el golfo de Guayaquil” por Lorenzo et al. Boletín de Geología, 41(2), 151-157. DOI: 10.18273/revbol.v41n2-2019009

Queremos aprovechar la oportunidad para felicitar la contribución de Lorenzo et al. (2018) así como su interés y esfuerzo en aportar soluciones a uno de los enigmas más importantes del sistema petrolero del Suroeste ecuatoriano. Durante las últimas tres décadas, hemos tenido la oportunidad de integrar estudios geológicos, geofísicos y geoquímicos en nuestro afán de no sólo alcanzar una interpretación regional coherente, sino también con el propósito de revelar y documentar los posibles sistemas petroleros de la Cuenca Progreso s.l. y su faja plegada. El propósito de esta exégesis es adicionar información de muchos reportes y estudios que se encuentran en la base de datos de PetroProducción, Petroamazonas (Belco Petroleum Ecuador, 1988, 1991; Marksteiner y Alemán, 1991; Alemán, 2000; PDVSA, 2011; PDVSA, 2014a, 2014b), que de un modo u otro ayudará a reforzar lo expresado en el trabajo de Lorenzo et al. (2018).

Un punto interesante a profundizar en este último trabajo de Lorenzo et al. (2018) está asociado a la interpretación geológica de la roca madre en relación a las vías de migración. En nuestra opinión, los crudos de la Península y la del Golfo de Guayaquil, incluyendo los campos de Albacora y Zorritos tienen una misma roca madre que es la Formación Dos Bocas y su equivalente en el Perú (Formación
Heath). Las sutiles diferencias geoquímicas que reportan Lorenzo et al. (2018) y que los invita a definir dos familias de crudos son consistentes con una misma unidad litológica que es la Formación Dos Bocas. La presencia de estas dos familias en una misma formación se debe no solo a los cambios de facies de la roca madre (presencia localizada de diatomitas y lutitas ricas en foraminíferos calcáreos), sino también a la distancia, grado de tortuosidad y capas trasportadoras (carrier beds) que atraviesan los crudos durante los procesos de migración desde la cocina generativa a los diversos campos, así como a la presencia de dos cocinas muy distintas que alimentan estos campos.

Por razones históricas, es justo y de vital importancia dar crédito a los esfuerzos titánicos del antiguo laboratorio de PetroProducción en Guayaquil que con escasos recursos analizaron todas las posibles rocas madres (CEPET-SRG, 1986; Litton Resources Group, 1986) lo que facilitó la decisión para que otras compañías exploradoras y consultoras enfocaran sus esfuerzos para discriminar en la columna estratigráfica las posibles unidades con potencial generativo (FIGURA 1). Gracias a ello, se llegó a descartar el bajo Contenido Orgánico Total (COT) de las calizas cineríticas silicificadas de la Formación Guayaquil que

\footnotetext{
1 GPG Services, Quito, Ecuador. (*) antenor.aleman@gpgservicesec.com, allly.palencia@gpgservicesec.com, eduardo.lezama@gpgservicesec.com

${ }^{2}$ Laboratorio de Mineralogía Óptica y Petrografía, Escuela Superior Politécnica del Litoral, Guayaquil, Ecuador. gmontene@espol.edu.ec
} 
localmente presentan una estratificación rítmica. También las lutitas de la Formación Estancia del Grupo Azúcar resultaron ser muy pobres en COT, al igual que se ignoró la importancia de los delgados carbones transportados del Grupo Ancón. Por otro lado, también se descartó las facies locales de lutitas no bioturbadas con alto COT de la Formación San Eduardo, no solo por su distribución muy local sino también por el hecho que esta unidad nunca se ha reportado en subsuelo. Como resultado de estos estudios se llegó a restringir el posible potencial generativo a dos unidades litológicas: La Formación Calentura del Turoniense y la Formación Dos Bocas/Heath del Mioceno Temprano a Medio (FIGURA 1).

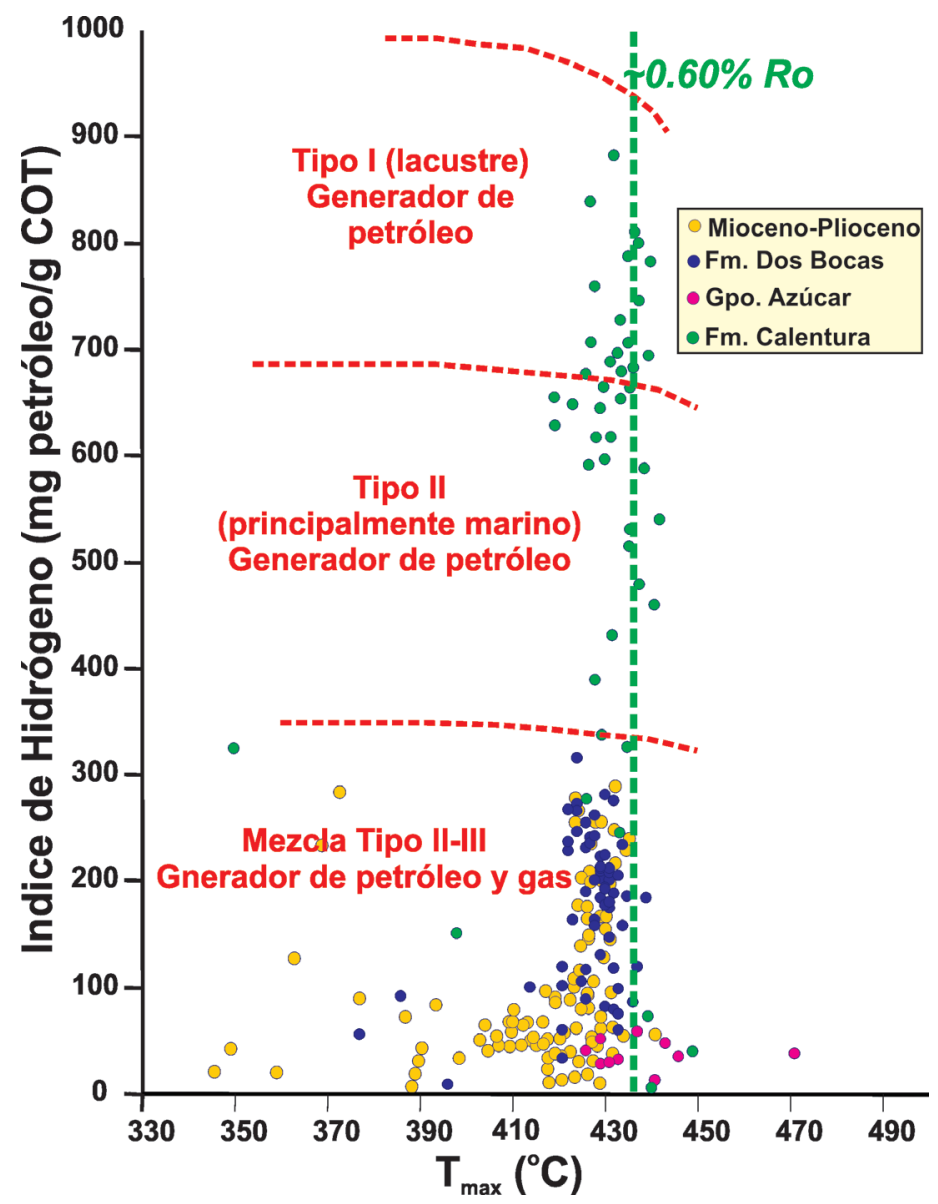

FIGURA 1. Diagrama que compara el Índice de Hidrógeno con el $\mathrm{T}_{\max }$ de las posibles rocas madres del suroeste ecuatoriano y en la cual se ilustra el bajo grado de madurez de casi todas las unidades estratigráficas investigadas (integración de data de Litton Resources Group, 1986; Alemán, 2000; GEMS, 2007).

El Evento Anóxico Oceánico (OAE2) de la Formación Calentura del Turoniense (Turgeon y Brumsack, 2006; Westermann et al., 2010) se mantuvo por mucho tiempo como un paradigma para explicar la roca madre de los crudos de la Península. Esta idea tomó fuerza durante el trabajo exploratorio de Belco (1988), quien proponía una cocina generativa al norte de la Península de Santa Elena basados en la presencia de hidrocarburos en el Pozo Dorado 1X. Desafortunadamente, aunque los horizontes de calizas negras de esta formación tienen hasta $8 \%$ de COT; estas no solo son relativamente muy delgadas sino que también están interestratificadas con tobas de ceniza y lapilli así como lutitas cineríticas altamente silicificadas que de ningún modo podrían comportarse como capas transportadoras ("carrier beds"). Desde el punto de vista de migración, su validez como roca madre empeora aún más. Es así, que sobreyaciendo a la Formación Calentura existen más de $2.000 \mathrm{~m}$ de brechas piroclásticas y hyaloclásticas lavas basálticas y andesitas basálticas interestratificadas con potentes capas de tobas de lapilli y lutitas cineríticas altamente silicificadas encima que imposibilitarían cualquier migración de hidrocarburos. Igualmente importante es que, además de no existir una correlación 
entre los crudos y una roca madre Cretácica, hasta el momento no se ha reportado esta unidad en subsuelo y si acaso existiera, su migración sería muy tortuosa e ineficiente.

Dadas estas consideraciones negativas de la Formación Calentura como posible roca madre, un cambio de paradigma era necesario para continuar explorando en el suroeste ecuatoriano. Inicialmente, la mayor parte de muestras de afloramiento de la Formación Dos Bocas tenían un COT menor de 1\% debido a la oxidación de la materia orgánica, excepto los afloramientos de esta formación en la Playa de Ayangue. Esto cambió dramáticamente cuando se obtuvieron valores más altos de COT al analizar la Formación Dos Bocas en los "coreholes" de Rodeo (Alemán, 2000). Es así que los perfiles de COT e Índice de Hidrógeno $\left(\mathrm{S}_{2} / \mathrm{gCOT}\right)$ de estos núcleos ("coreholes") muestran que existe un gran potencial de generación para la Formación Dos Bocas, la cual alcanza valores promedio de COT de $3 \%$ en un intervalo de más de 200 m (FIGURA 2).

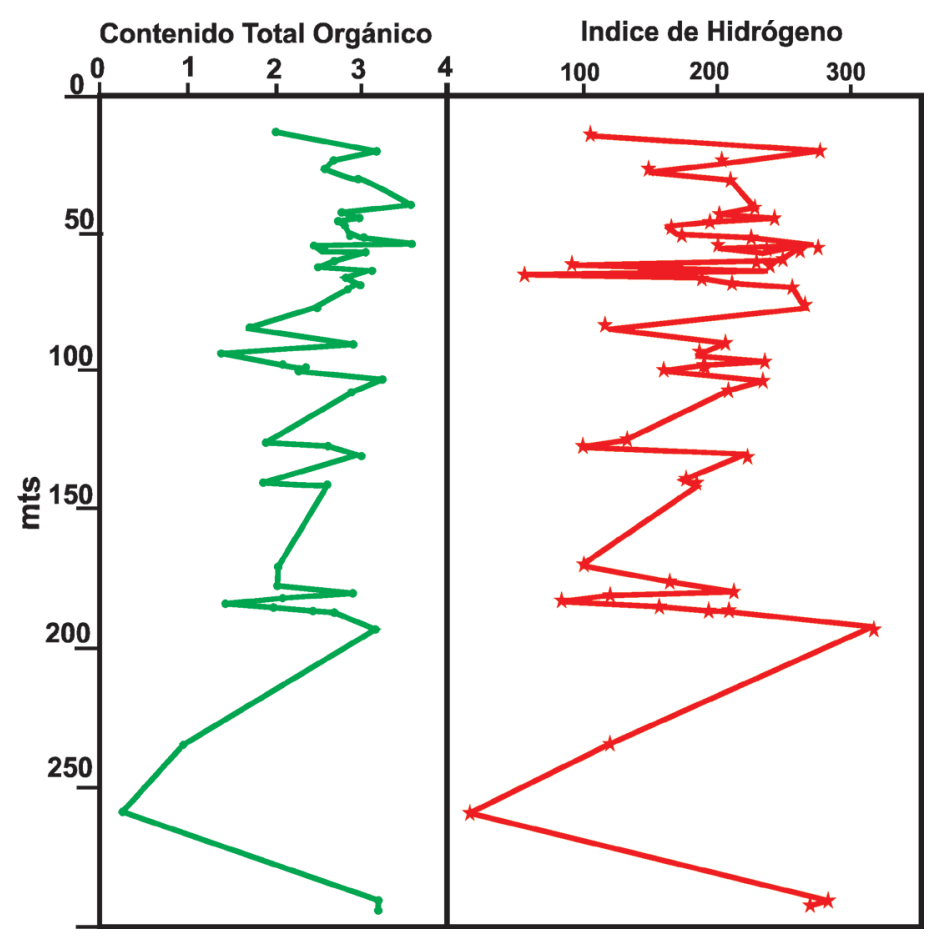

FIGURA 2. Registro compuesto de los núcleos de la Formación Dos Bocas en los “coreholes” de Rodeo. Tomado de Alemán (2000).

Estudios adicionales de correlación de roca madre y petróleo permitieron confirmar que tanto la Formación Dos Bocas como su equivalente la Formación Heath en el Perú, son las rocas madre, no solo de los crudos de los campos de Albacora y Zorritos en el Perú sino también del petróleo recuperado en el pozo Golfo de Guayaquil-1 en Ecuador (Alemán, 2000; GEMS, 2007; Lorenzo et al., 2018). La sorpresa más agradable fue al documentar también que los pozos Pacoa-1X y Golfo de Guayaquil-1 tenían un alto contenido de oleonanos y sus cromatogramas eran muy similares a los crudos del Golfo de Guayaquil (Alemán, 2000). Más tarde, en un estudio encomendado a GEMS (2007), los análisis de biomarcadores corroboran la importancia de la Formación Dos Bocas como roca madre. Nuestros estudios regionales nos permiten re-interpretar la posible presencia geoquímica de diferentes "familias de crudos" (Lorenzo et al., 2018) en términos de cambios de facies y cocinas diferentes. Evidencias de campo y descripciones de subsuelo sugiere la presencia de varios pulsos de "upwelling" documentadas en las diatomitas de las Facies Villingota, la recurrencia de lutitas con un alto contenido de foraminíferos calcáreos así como la presencia siliciclástios gruesos asociados a los procesos de extensión y apertura de la Cuenca Progreso s.s. (PDVSA, 2014b).

Igualmente importante ha sido la documentación de patrones idénticos y en particular la presencia del pico de Oleonano (m/z191 Terpanos Pentacíclicos) en los fragmentogramas de los crudos de los pozos: Guayacan-1, Palo Santo-1X, Pacoa-1X, Centinela-1X, 
San Pablo-1X y La Matachivatos- 1X que fuertemente sugieren un aporte de una roca Terciaria (GEMS, 2007; PDVSA, 2014a). Esto también se confirma en el gráfico de la Relación Oleonano/Hopano versus Ganmacerano/C30Hopano que sugiere una asociación a una roca madre Terciaria para todas las muestras de crudo (GEMS, 2007; PDVSA, 2014a, 2014b).

No hay duda sobre la fuerte correlación de los biomarcadores saturados para los extractos de roca y crudos de la Cuenca Progreso y Golfo de Guayaquil. Esta correlación nos muestra una robusta correlación entre los extractos de La Formación Dos Bocas con el crudo del pozo Golfo de Guayaquil-1 y su continuación hacia los campos de Albacora y Zorritos en Perú. Los isótopos de carbón (13C) de las fracciones aromáticas y saturadas de los diferentes extractos y crudos no solo corroboran una tendencia hacia un origen marino (FIGURA 3) sino que también resaltan la similitud de la huella isotópica del extracto de la Formación Dos Bocas con los crudos del pozo Golfo de Guayaquil-1 y Campo Zorritos en Perú y algunos campos de la Península de Santa Elena. Los pozos de la Península que difieren isotópicamente se explican no solo en términos de cambios de facies sino también desde el punto de vista de su historia migratoria, como se mencionó anteriormente (Alemán, 2000; PDVSA, 2011). Más aun, el diagrama ternario composicional SARA (Saturados, Aromáticos, Resinas y Asfáltenos), de varios crudos de la Península de Santa Elena, Campo Pacoa y el pozo Golfo de Guayaquil-1 no solo muestra una gran similitud de estos crudos sino que también indica la presencia de una alta proporción de hidrocarburos saturados, a pesar de la evidencia de procesos de biodegradación que han afectado a los hidrocarburos de la Península de Santa Elena (FIGURA 4), como también lo indica Lorenzo et al. (2018).

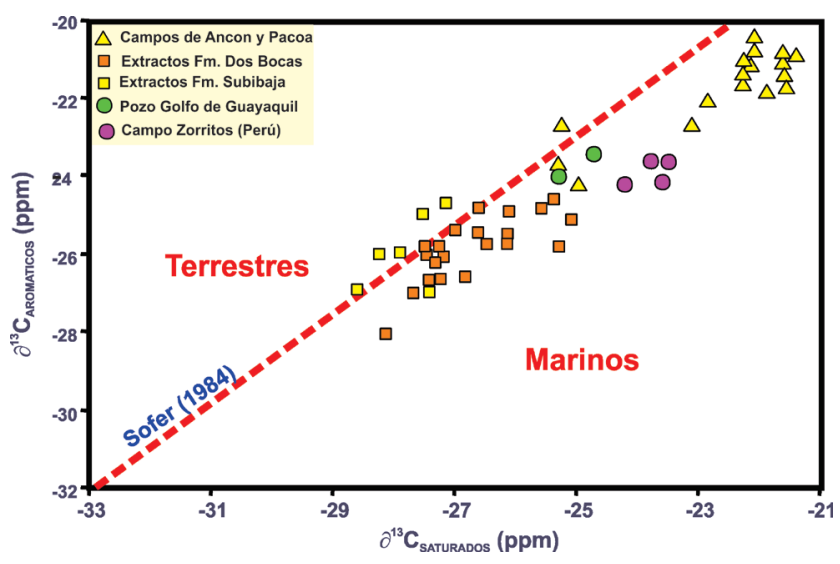

FIGURA 3. Gráfico de Sofer (1984) de los isótopos de la fracción aromática versus la fracción saturada para diferentes tipos de crudos incluyendo los de la Península de Santa Elena, Golfo de Guayaquil y el Campo Zorritos en el Perú así como los extractos de la Formación Dos Bocas y pozos del Golfo de Guayaquil (integración de data de Litton Resources Group, 1986; Alemán, 2000; GEMS, 2007; Lorenzo et al., 2018).

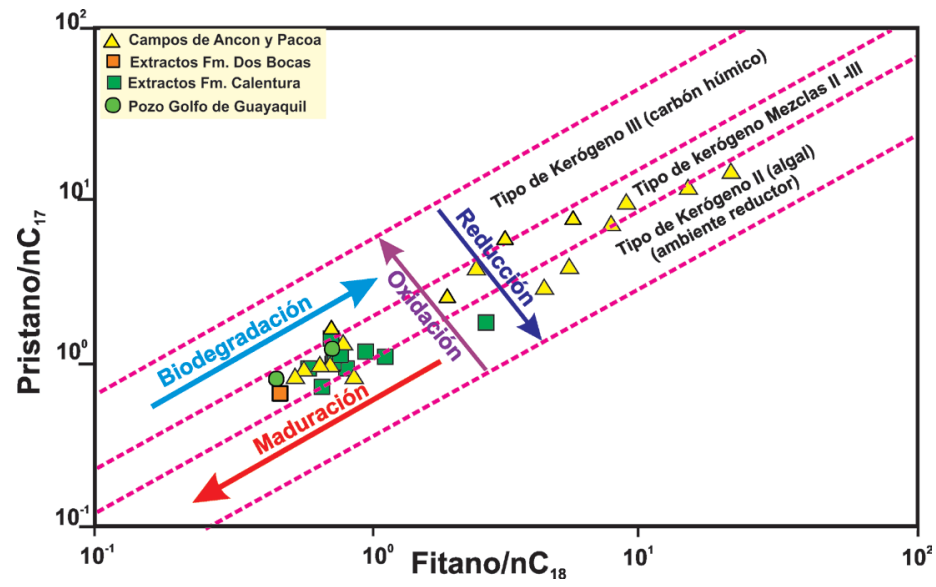

FIGURA 4. Relación Pristano/ $\mathrm{nC}_{17} v s$ Fitano/ $\mathrm{nC}_{18}$ que ilustra la gran variabilidad del grado de madurez y biodegradación de los crudos y que a su vez sugiere una depositación de la roca madre en un ambiente transicional en condiciones poco reductoras (tomado de GEMS, 2007, con datos de Alemán, 2000; Lorenzo et al., 2018). 


\section{MIGRACIÓN DEL CRUDO DESDE LA COCINA FORMACIÓN DOS BOCAS HACIA LA PENÍNSULA DE SANTA ELENA}

Nuestros estudios regionales sugieren la presencia de dos potenciales depocentros que podrían operar como cocinas generativas en el Golfo de Guayaquil (FIGURA 5). Sin embargo, el gran dilema es poder explicar la misma roca madre (Formación Dos Bocas) para el petróleo en los campos de la Península de Santa Elena donde esta unidad está ausente o aflorando. Igualmente importante es tener en consideración las siguientes evidencias.

1. El gradiente geotérmico de la Península de Santa Elena es menos de $2,2^{\circ} \mathrm{C} / 100 \mathrm{~m}$ lo que significa que todos los campos a menos de 2.200 m están en proceso de biodegradación.

2. La presencia de un evento erosivo de por lo menos $800 \mathrm{~m}$ documentado en los estudios de vitrinita de los pozos perforados por Belco Petroleum Ecuador (1988), tal como se observa en el pozo NSX-1X.

3. La ocurrencia de crudos de alto API que geoquímicamente es una mezcla de crudos biodegradados y no biodegradados en la Península de Santa Elena (Alemán, 2000; GEMS, 2007; Lorenzo et al., 2018). Esta mezcla, implica la presencia de una migración activa desde una cocina generativa activa que está en la ventana de petróleo. La variabilidad en el contenido de saturados de los campos de la Península de Santa Elena, no solo indica cierto grado de biodegradación sino también distintos pulsos de llenado hasta un proceso de llenado actual.

4. La alta proporción de hidrocarburos saturados, en crudos con evidencia de biodegradación, en la caracterización composicional SARA (Saturados, Aromáticos, Resinas y Asfáltenos), de varios pozos de la península, el Campo Pacoa y el Golfo de Guayaquil (FIGURA 4). Esta coexistencia una vez más sugiere fuertemente una recarga constante desde una cocina generativa activa. Esto se ilustra muy bien en el gráfico de la relación entre los C29-25 NorHop/ C30Hop vs ${ }^{\circ}$ API para la mayoría de los crudos de la Península de Santa Elena (GEMS, 2007).

5. El alto contenido de oleonanos en los crudos de la Península de Santa Elena sugiere una roca madre de edad Terciaria cuando las angiospermas alcanzaron su más alto desarrollo.

6. Los petróleos se correlacionan mejor con los extractos Terciarios. Este gráfico además sugiere que los crudos de la península han sido generados por una roca de mezcla de siliciclástios y carbonatos muy común en la Formación Dos Bocas en la que la fracción carbonática es el resultado de la abundancia de fauna en las lutitas.

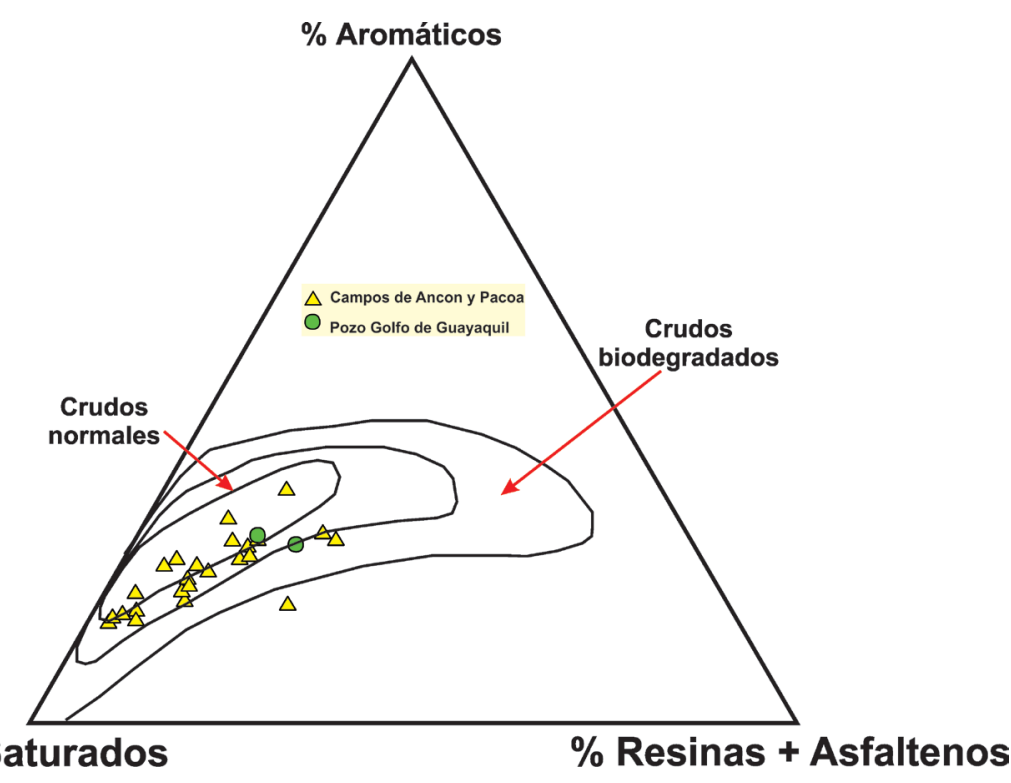

FIGURA 5. Diagrama triangular de composición SARA para los crudos de la Península de Santa Elena y Golfo de Guayaquil (tomado de GEMS, 2007; Alemán, 2000; Lorenzo et al., 2018). 
La roca madre de las dos cocinas generativas aunque tienen la misma unidad litológica, están caracterizadas por procesos tectónicos y ambientes depositacionales muy distintos que podrían explicar las dos posibles familias de crudos descritas desde el punto de vista geoquímico (Lorenzo et al., 2018). En la parte más al sur de la cuenca, la Formación Dos Bocas está caracterizada por facies lutáceas marinas con continua conexión al mar abierto y en la que los procesos de reducción y productividad biológica es menor a la ubicada al norte de la Isla Puna. Esta cocina del sur está alimentando y carga los campos de Amistad y Albacora a través de la Falla Guayaquil que se ha comportado intermitentemente como vía de migración y como sello en tiempo y espacio (Alemán, 2000). El campo Zorritos por otro lado, es alimentado por una cocina muy local asociada al bloque caído de la Falla Tronco Mocho.

La segunda cocina está ubicada en la parte más profunda de la Cuenca Progreso s.s. al norte de la Isla Puná. En esta cocina, la Formación Dos Bocas fue depositada en una cuenca restringida limitada por las fallas La Cruz y Carrizal formadas durante la apertura de la cuenca. En esta cuenca tipo desgarre ("pull-apart"), la productividad fue alta y con frecuencia se depositaron secuencias diatomáceas que sugieren procesos de "upwelling". Así mismo, como consecuencia de la extensión existen muchos aportes de siliciclástios contemporánea con la depositación (PDVSA, 2011). Es así que quizás existan sutiles diferencias geoquímicas que conlleva a definir los dos tipos de familias de hidrocarburos (Lorenzo et al., 2018). En nuestra opinión, esta cocina estaría alimentando los campos de la Península de Santa Elena a través de vías de migración establecidas por el Riedel de Rodeo, el sistema de fallas extensionales de la Falla La Cruz, las cuales enfocaron la migración hacia el Alto estructural creado en la Península de Santa Elena durante la deformación contractional del Eoceno (FIGURA 5). La documentación sísmica, tanto el Riedel de Rodeo como el de Playas por el equipo de PDVSA fue de vital importancia para poder comprender la presencia de los crudos de la Península de Santa Elena (PDVSA, 2011, 2014a, 2014b). Una vez que la migración llega a este alto, los hidrocarburos son transportados y acumulados principalmente en las arenas superiores del Grupo Azúcar conocidas localmente como Formación Atlanta. Estas arenas a su vez funcionan como "carrier beds", y a través de fallas, los hidrocarburos migraron hacia reservorios superiores del Grupo Ancón (FIGURA 6).

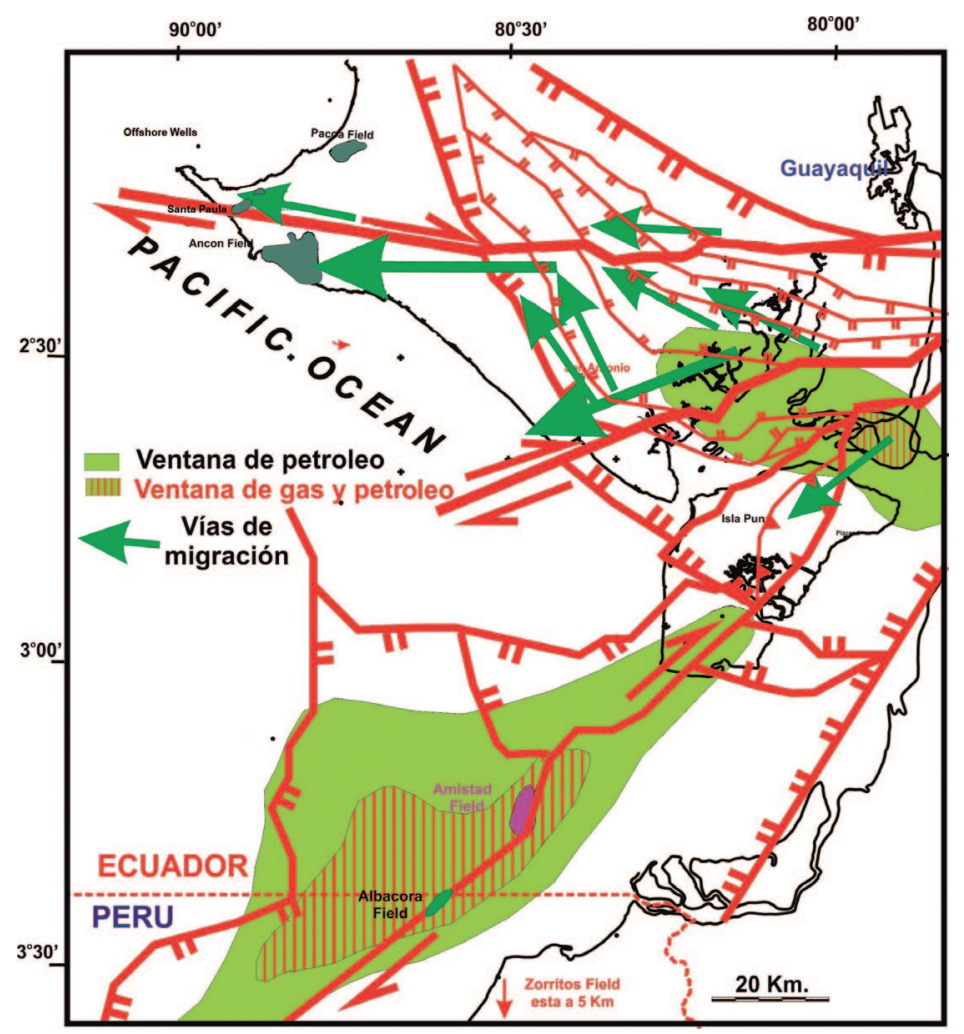

FIGURA 6. Cocinas generativas de la Cuenca Progreso s.l. con las posibles vías de migración que explicaría la correlación entre los crudos de los campos de la Península y los extractos de la Formación Dos Bocas. Observar que tanto el Campo Amistad como el Campo Albacora en la frontera Perú-Ecuador están ubicados a lo largo de la Falla Guayaquil. 
La migración de hidrocarburos continuó hacia el norte de la Península de Santa Elena, pero cada vez que saltaba a otra estructura se hacía más tortuosa y menos eficiente (PDVSA, 2014a, 2014b).

Como consecuencia de la poca profundidad de los reservorios y el bajo gradiente térmico, los hidrocarburos migrados y entrampados en trampas estructurales fueron y están siendo biodegradados. Sin embargo, el hecho de tener una cocina generativa activa y la migración continua hasta el presente, es posible encontrar en los campos de la Península de Santa Elena, una mezcla de crudos biodegradados y no biodegradados.

\section{REFERENCIAS}

Alemán, A.M. (2000). Technical Evaluation Agreement of the Progreso Basin.

Belco Petroleum Ecuador. (1988). Revisión Geológica y Potencial Petrolero de la Región Costa Afuera al Sur de la Península de Santa Elena, Ecuador.

Belco Petroleum Ecuador. (1991). Sumario de perforación Pozo Mata Chivato-X1. Santa Elena, Ecuador.

CEPET-SRG. (1986). Evaluación de la Cuenca Progreso.

GEMS. (2007). Evaluación de los sistemas petrolíferos de las Cuencas del Suroeste del Litoral Ecuatoriano. Informe Final.

Litton Resources Group. (1986). The Gulf of Guayaquil Basin Study; Corporación Estatal Petrolera Ecuatoriana Report.

Lorenzo, E., Roca-Beltrán, W.A., Martínez, M., Morato, A., Escandón-Panchana, P.C., y ÁlvarezDomínguez, C.P. (2018). Correlación geoquímica entre crudos y rocas del sistema petrolero de la península de Santa Elena y el golfo de Guayaquil. Boletín de Geología, 40(1), 31-42. doi: 10.18273/ revbol.v40n1-2018002.
Marksteiner, R., and Alemán, A.M. (1991). Coastal Ecuador Technicial Evaluation Agreement, AMOCO Prod. Co y PETROECUADOR, 218 p.

PDVSA. (2011). Informe Final Proyecto Ecuador, Bloque 4.

PDVSA. (2014a). Informe Final Proyecto Ecuador, Bloque 1.

PDVSA. (2014b). Informe Final Proyecto Ecuador, Bloque 5.

Sofer, Z. (1984). Stable carbon isotope compositions of crude oils: Application to source depositional environments and petroleum alteration. $A A P G$ Bulletin, 68(1), 31-49. doi: 10.1306/AD46096316F7-11D7-8645000102C1865D.

Turgeon, S., and Brumsack, H.J. (2006). Anoxic vs dysoxic events reflected in sediment geochemistry during the Cenomanian-Turonian Boundary Event (Cretaceous) in the Umbria-Marche Basin of central Italy. Chemical Geology, 234(3-4), 321339. doi: 10.1016/j.chemgeo.2006.05.008.

Westermann, S., Caron, M., Fiet, N., Fleitmann, D., Matera, V., Adatte, T., and Föllmi, K.B. (2010). Evidence for oxic conditions during oceanic anoxic event 2 in the northern Tethyan pelagic realm. Cretaceous Research, 31(5), 500-514. doi: 10.1016/j.cretres.2010.07.001.

\begin{tabular}{c}
\hline Antenor M. Alemán \\
ORCID: 0000-0003-2585-198X \\
Galo Montenegro \\
ORCID: 0000-0002-5976-2179 \\
Allly Palencia \\
ORCID: 0000-0001-7067-1748 \\
Eduardo Lezama \\
ORCID: $0000-0002-9439-5546$ \\
\hline \hline
\end{tabular}

Trabajo recibido: septiembre 03 de 2018

Trabajo aceptado: diciembre 12 de 2018 